\title{
Tuberculous Fibrosis of Lung
}

National Cancer Institute

\section{Source}

National Cancer Institute. Tuberculous Fibrosis of Lung. NCI Thesaurus. Code C35088.

Scarring of the lung parenchyma caused by pulmonary tuberculosis. 